\title{
Real-world effectiveness of admissions to a tertiary treatment-resistant psychosis service: 2-year mirror-image study
}

Cecilia Casetta, Fiona Gaughran, Ebenezer Oloyede, Juliana Onwumere, Megan Pritchard, Sukhi S. Shergill, Eromona Whiskey and James Hunter MacCabe

\section{Background}

Treatment-resistant schizophrenia is a major disabling illness which often proves challenging to manage in a secondary care setting. The National Psychosis Unit (NPU) is a specialised tertiary in-patient facility that provides evidence-based, personalised, multidisciplinary interventions for complex treatment-resistant psychosis, in order to reduce the risk of readmission and longterm care costs

\section{Aims}

This study aimed to assess the long-term effectiveness of treatment at the NPU by considering naturalistic outcome measures.

\section{Method}

Using a mirror image design, we compared the numbers of psychiatric and general hospital admissions, in-patient days, acuity of placement, number of psychotropic medications and dose of antipsychotic medication prescribed before and following NPU admission. Data were obtained from the Clinical Records Interactive Search system, an anonymised database sourced from the South London and Maudsley NHS Trust electronic records, and by means of anonymous linkage to the Hospital Episode Statistics system.

\section{Results}

Compared with the 2 years before NPU admission, patients had fewer mental health admissions $(1.65 \pm 1.44 v .0 .87 \pm 0.99$,
$Z=5.594, P<0.0001)$ and less mental health bed usage (335.31 \pm 272.67 v. $199.42 \pm 261.96, Z=5.195 P<0.0001)$ after NPU admission. Total in-patient days in physical health hospitals and total number of in-patient days were also significantly reduced $(16.51 \pm 85.77$ v. $2.83 \pm 17.38, Z=2.046, P=0.0408 ; 351.82 \pm$ $269.09 v .202 .25 \pm 261.05, Z=5.621, P<0.0001)$. The reduction in level of support required after treatment at the NPU was statistically significant $(z=-8.099, P<0.0001)$.

\section{Conclusions}

This study demonstrates the long-term effectiveness of a tertiary service specialising in treatment-resistant psychosis.

\section{Keywords}

Treatment-resistant psychosis; tertiary service; personalised care; clozapine; specialist service.

\section{Copyright and usage}

(C) The Author(s), 2020. Published by Cambridge University Press on behalf of the Royal College of Psychiatrists. This is an Open Access article, distributed under the terms of the Creative Commons Attribution-NonCommercial-NoDerivatives licence (http://creativecommons.org/licenses/by-nc-nd/4.0/), which permits non-commercial re-use, distribution, and reproduction in any medium, provided the original work is unaltered and is properly cited. The written permission of Cambridge University Press must be obtained for commercial re-use or in order to create a derivative work.
Schizophrenia is a major disabling illness which affects approximately $1 \%$ of the population worldwide ${ }^{1}$ and contributes 13.4 million years lived with disability to the global burden of disease. ${ }^{2}$ Approximately one-third of patients with a schizophrenia spectrum diagnosis have treatment-resistant schizophrenia (TRS), ${ }^{3}$ i.e. they fail to respond to adequate trials of two different antipsychotics. ${ }^{4}$

\section{Tertiary services for complex TRS}

Patients with TRS often have severe and enduring health needs; some are particularly difficult to manage in primary or secondary care and require a higher level of support. ${ }^{6}$ In the UK, tertiary care is defined as individualised and specialised multidisciplinary interventions delivered by highly trained staff in order to address problems that are complex and refractory to standard interventions. ${ }^{7}$ Tertiary services focus on high-intensity treatment of a relatively small number of the most difficult-to-treat individuals ${ }^{8}$ and offer specialist expertise with a high staff/patient ratio. Tertiary care programs for TRS should include individually tailored psychosocial rehabilitation, as well as personalised and evidence-based medication management, to achieve flexible and effective care. ${ }^{9}$

Clozapine is the only recommended antipsychotic treatment for TRS and is the most effective agent for reducing symptoms, ${ }^{10}$ hospital use, ${ }^{11}$ suicide rates, ${ }^{12,13}$ aggressive behaviour, ${ }^{14}$ violent crime ${ }^{15}$ and substance misuse. ${ }^{16}$ However, the management of clozapine therapy for patients with severe and chronic behavioural problems requires carefully developed strategies and close monitoring of adverse effects, which can be challenging in a primary or secondary care setting. Clozapine has been found to be largely underutilised by prescribing clinicians, ${ }^{17}$ with a considerable number of TRS patients being left on high-dose antipsychotics or polypharmacy, ${ }^{18}$ despite neither of these options having a good evidence base. ${ }^{19,20}$

\section{The National Psychosis Unit}

The National Psychosis Unit (NPU) seeks to provide specialist evidence-based treatment for people with treatment-refractory psychosis, in order to reduce the risk of readmission and expensive longterm care costs. It is part of the National Psychosis Service (NPS), which produces out-patient and outreach assessments aimed at providing specialist input into the management of complex and refractory psychosis. The NPS is open to referrals from across the UK and beyond, and it offers an in-patient service to those patients who are deemed to need longer-term multidisciplinary and specialised input.

The NPU is an 18-bed mixed-gender in-patient facility situated within the Bethlem Royal Hospital and forms part of the South London and Maudsley (SLaM) NHS Trust. The NPU multidisciplinary team consists of psychiatrists, psychiatric nurses, pharmacists, social workers, and allied health professionals including 
occupational therapists and clinical psychologists. Furthermore, the NPU has an established close partnership with physical health physicians from King's Health Partners, including cardiologists and haematologists, enabling multidisciplinary medical discussions and support for the patients admitted.

Patients are referred to the NPU after treatments has failed to produce sufficient clinical improvement. Most patients are transferred from in-patient settings where they are detained under the Mental Health Act. They receive a comprehensive, multidisciplinary review of their previous psychiatric, medical and medication history, leading to an individualised care package which will typically include optimisation of pharmacological treatment and physical health, occupational therapy activities, social work input, and individual and family-based psychological interventions that have proven effective in reducing symptoms and distress and improving social functioning in TRS. ${ }^{21}$ Optimisation of clozapine treatment, the management of its side-effects, clozapine re-challenge after suspected myocarditis, episodes of neutropenia or agranulocytosis, gastro-intestinal obstruction or other potentially life-threatening adverse effects, and issues regarding non-adherence constitute a large proportion of the work of the NPU. Another key element is the multimorbidity approach, which includes proactive physical health promotion including smoking cessation, physical exercise and weight control.

Although we have previously demonstrated short-term positive outcomes in patients admitted to the NPU, ${ }^{22,23}$ we have not described the longer-term outcomes. This study aimed to quantify the long-term effectiveness of treatment at the NPU by considering naturalistic outcome measures. Using a mirror image design, we used anonymised data from electronic health records to compare the number of psychiatric and general hospital admissions, inpatient days, acuity of placement, number of psychotropic medications and dose of antipsychotic medication prescribed before and after the treatment at the NPU.

\section{Method}

\section{Data sources}

Data were obtained from the Clinical Records Interactive Search (CRIS) system. CRIS is a large, de-identified psychiatric database sourced from SLaM electronic health records. It protects patient anonymity and maximises the data available for research. Developed in 2007, the CRIS application provides researchers with both structured and unstructured (open text) data in anonymised form from the full clinical record. ${ }^{21}$

Age at admission to the NPU, gender, ethnicity, length of admission and date of death (when relevant) were obtained from CRIS structured fields.

Data on length of psychotic illness at admission, number of previous antipsychotic trials, acuity of residence before admission and after discharge, use of clozapine at admission and discharge, number of psychotropic medications, and antipsychotic medication dose at admission and discharge were manually collected from open text. The British National Formulary (BNF) total antipsychotic dosage was used to convert antipsychotic dosage into percentage of maximum recommended daily antipsychotic dose as per BNF guidelines. ${ }^{24}$

Data on psychiatric and general hospital admissions and Accident and Emergency (A\&E) admissions during the 2 years before and the 2 years after NPU treatment were obtained by means of an anonymous linkage to the Hospital Episode Statistics (HES; https://digital.nhs.uk/data-and-information/data-tools-andservices/data-services/hospital-episode-statistics) system, a data warehouse containing details of all admissions at National Health
Service (NHS) hospitals in England. The CRIS application is linked to the HES system; this ensures data anonymity.

\section{Data definitions}

This study used a mirror image design with the treatment at the NPU defined as the mirror. The period of 2 years prior to the date of admission to the NPU was used as the pre-mirror period, while a period of 2 years from the date of discharge was used as the postmirror period. Data on number of admissions to psychiatric and general hospitals and to $\mathrm{A} \& \mathrm{E}$ during the pre- and post-mirror periods were collected, together with admission and discharge dates.

Medications at admission and discharge for which data were collected included antipsychotics, anticholinergics, benzodiazepines, mood stabilisers and antidepressants; dosages for antipsychotics were also collected. Pro re nata prescriptions were not considered.

Data on placements immediately before and after the admission to the NPU were categorised as psychiatric intensive care unit (PICU), acute ward, rehabilitation service, care home, supported accommodation and independent living (including living with family). They were ordered from the highest to the lowest intensity and were given a number from 1 to 6 . The difference between preadmission and post-discharge acuity was calculated.

\section{Sample inclusion criteria}

The cohort consisted of all patients ever admitted to the NPU after 1 January 2007 and discharged before 31 March 2015. This study period was selected because electronic records were fully implemented in SLaM in 2007, and admissions with a discharge date before 31 March 2015 allowed a 2-year follow up on the HES system, since the latest HES update available at the time of the study had data until 31 March 2017.

The process of cohort identification is detailed in Fig. 1. A total of 160 patients were included in the demographical analysis. Three patients were excluded from the mirror-image study because they died within the post-mirror period, and ten patients did not have a match in the HES database or had unreliable data (e.g. outliers). A total of 147 patients admitted to the NPU were included in the mirror-image study. A sensitivity analysis was conducted to address the issue of missing data due to lack of information in the HES system about patients coming from outside England. Seven patients residing outside England were therefore excluded, and 140 patients were included in the sensitivity analysis.

\section{Statistical analysis}

Statistical analysis was carried out using Stata version 15. Number of admissions to general and psychiatric hospitals, presentation to A\&E, general, psychiatric and total in-patient days, and placements before and after treatment at the NPU were compared within patients between the mirror-image periods using a Wilcoxon signed-rank test, as the data were not normally distributed. Sensitivity analysis included only patients living in England. Within-subject comparisons of number of psychotropic medications and percentages of BNF maximum antipsychotic dose at admission and discharge were conducted using paired t-tests. Clozapine medication at admission and discharge from NPU was compared using $\chi^{2}$-test. Comparison between acuity of placement before and after the treatment at the NPU was carried out using Wilcoxon-signed rank test.

Finally, we performed multiple linear regressions with the change in psychiatric, general and total in-patient days before and after the mirror points as the dependent variables, and the following covariates: age at admission, gender, duration of psychotic illness, length of admission and initiation of clozapine during admission. 
Patients ever referred

and admitted to the NPU

(admitted after 1 January 2007 and discharged before 31 March 2015)

$n=165$

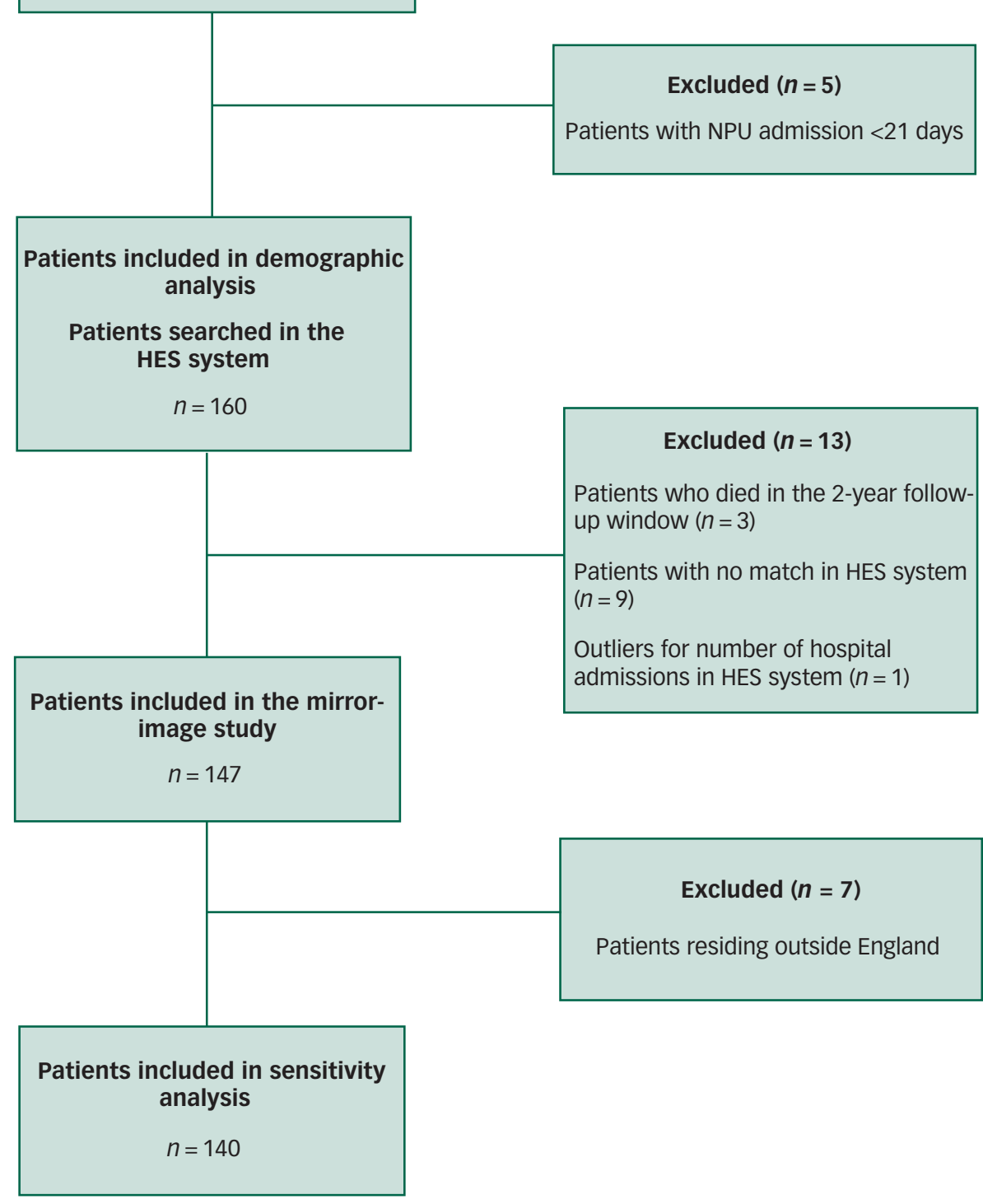

\section{Fig. 1 Cohort selection}

\section{Ethical approval}

Overarching ethical approval for the use of CRIS as a research dataset was given by Oxfordshire Research Ethics Committee C (08/ H0606/71), with individual projects approved by a patient-led oversight committee. This study was approved by the CRIS oversight committee (reference number 18-105). Informed consent was not required as CRIS is an anonymised case register.

\section{Results}

\section{Study population}

Table 1 summarises patient characteristics for the 160 admissions included in the demographic analysis.

\section{Hospital admissions and length of stay}

Patients had significantly fewer mental health admissions (1.65 \pm 1.44 v. $0.87 \pm 0.99, z=5.594, P<0.0001)$ and shorter total length of stay $(335.31 \pm 272.67$ v. $199.42 \pm 261.96, z=5.195, P<0.0001)$ in the 2 year period after treatment at the NPU, compared with the 2 years before admission. Similarly, total days of physical health admissions was significantly reduced $(16.51 \pm 85.77$ v. 2.83 $\pm 17.38, z=2.046, P=0.0408$ ) (Fig. 2). There was also a reduction in the number of physical health admissions $(0.59 \pm 2.03 v . .31 \pm$ $1.07)$, although this was of borderline statistical significance $(z=$ $1.959, P=0.0501)$, while the reduction in A\&E presentations (2.09 \pm 7.54 v. $1.83 \pm 7.00)$ was not statistically significant $(z=-0.374$, $P=0.7081)$. Total number of in-patient days was significantly reduced after the admission compared with before (351.82 \pm 269.09 v. $202.25 \pm 261.05, z=5.621, P<0.0001)$. Results for all 


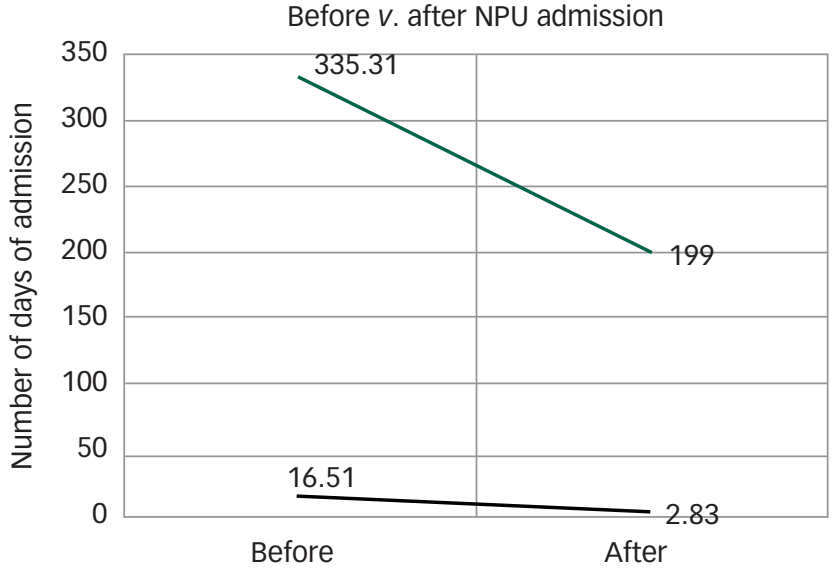

_ Mental health admissions

General admissions

Fig. 2 Mean days of admission to mental health and general hospitals in the 2 years before versus 2 years after the NPU admission.

\begin{tabular}{|lc}
\hline Table $1 \quad$ Characteristics of patients admitted to the NPU & \\
\hline Characteristics & Mean \pm S.d. \\
\hline Female gender ( $\mathrm{n}, \%)$ & $75(47)$ \\
Age at admission (years) & $35.24 \pm 11.89$ \\
Primary ICD diagnosis (n, \%) & \\
F20 - Schizophrenia & $85(58)$ \\
F25 - Schizoaffective disorder & $39(27)$ \\
F29 - Unspecified psychosis & $2(1)$ \\
F31 - Bipolar disorder & $8(5)$ \\
F32 - Depressive disorder & $2(1)$ \\
F70 - Mental retardation & $4(3)$ \\
Other diagnosis & $6(4)$ \\
Ethnicity (n, \%) & \\
White & $109(69)$ \\
Black African & $22(14)$ \\
Black Caribbean & $10(7)$ \\
Other ethnicity & $16(10)$ \\
Length of illness (years) & $12.92 \pm 8.85$ \\
Length of admission to NPU (days) & $322.53 \pm$ \\
& 200.93 \\
Number of previous different antipsychotic trials & $5.66 \pm 2.37$ \\
Patients in hospital consistently for $\geq 2$ years prior to NPU & $21(13)$ \\
admission (n, \%) & \\
a. Other diagnoses include: autism spectrum disorder, emotionally unstable personality \\
disorder, organic psychosis, delusional disorder, dissociative disorder, substance- \\
induced psychosis.
\end{tabular}

the variables investigated were robust to the sensitivity analyses. Table 2 summarises the outcome measures considered in the study.

\section{Polypharmacy and antipsychotic dosage}

No statistical difference was found in the numbers of psychotropic medications prescribed at admission and discharge $(2.79 \pm 1.28 v$. $2.91 \pm 1.38, \quad t=-0.9846, \quad P=0.8367)$. The percentage of BNF maximum antipsychotic dose was significantly higher at admission to the NPU compared with at discharge $(87.25 \pm 52.21 v .73 .52 \pm$ $44.16, t=2.6289, P=0.0048$ ). A higher proportion of patients were taking clozapine at discharge compared with at admission (95 v. 25, Pearson's $\chi^{2}=12.3900, P<0.0001$ ).

\section{Acuity of placement}

Patients' placements before admission to and after discharge from the NPU were compared. A value from 1 to 6 was assigned to each placement, from the highest (PICU) to the lowest (independent living) intensity. The reduction in level of support before and after treatment at the NPU was statistically significant $(z=$ $-8.099, P<0.0001)$. Figure 3 shows a mosaic plot of patients' placements pre- and post-NPU treatment, demonstrating that the majority of patients were admitted from acute in-patient units and most were discharged to rehabilitation units or independent living.

\section{Predictors of good outcome}

In the multiple linear regression, age at admission, gender, ethnicity, duration of psychotic illness, length of admission and introduction of clozapine were not associated with any statistically significant reduction in total in-patient days in psychiatric hospitals $\left(F(5131)=1.28, P=0.28, R^{2}=0.05\right)$, general hospitals $(F(5131)=1.02$, $\left.P=0.41, R^{2}=0.04\right)$ or psychiatric and general hospitals combined $\left(F(5131)=1.52, P=0.19, R^{2}=0.05\right)$.

\section{Discussion}

This study aimed to investigate long-term outcomes of treatment at a specialised in-patient facility for adults with a treatment-resistant psychotic disorder. Numbers of psychiatric admissions and psychiatric, general and overall in-patient days were lower in the 2 years following the intervention than in the 2 years prior.

These results are in line with those of previous studies of mental health tertiary services, which have evaluated specialised facilities dedicated to affective disorders. ${ }^{25-27}$ In fact, diseasespecific integrated care models are becoming more common, especially for long-term conditions such as epilepsy; by specialising in a particular clinical area, centres of excellence can develop targeted

\begin{tabular}{|c|c|c|c|c|}
\hline & $\begin{array}{l}\text { Two years pre-NPU } \\
\text { admission (mean } \pm \text { S.d.) }\end{array}$ & $\begin{array}{l}\text { Two years post-NPU } \\
\text { admission (mean } \pm \text { S.d.) }\end{array}$ & $P$-value & $\begin{array}{l}P \text {-value for } \\
\text { sensitivity } \\
\text { analyses }^{a}\end{array}$ \\
\hline Number of mental health admissions & $1.66 \pm 1.44$ & $0.87 \pm 0.99$ & $P<0.0001$ & $P<0.0001$ \\
\hline Total days of mental health admissions & $335.31 \pm 272.67$ & $199.42 \pm 261.96$ & $P<0.0001$ & $P<0.0001$ \\
\hline Number of physical health admissions & $0.59 \pm 2.03$ & $0.31 \pm 1.07$ & $P=0.0501$ & $P=0.0500$ \\
\hline Total days of physical health admissions & $16.51 \pm 85.77$ & $2.83 \pm 17.38$ & $P=0.0408$ & $P=0.0407$ \\
\hline Number of A\&E admissions & $2.10 \pm 7.54$ & $1.83 \pm 7.10$ & $P=0.7081$ & $P=0.7095$ \\
\hline $\begin{array}{l}\text { Total number of in-patient days (mental health admissions + } \\
\text { physical health admissions) }\end{array}$ & $351.82 \pm 269.10$ & $202.25 \pm 261.05$ & $P<0.0001$ & $P<0.0001$ \\
\hline Number of psychotropic medications (admission $v$. discharge) & $2.79 \pm .28$ & $2.91 \pm 1.38$ & $P=0.8367$ & $\mathrm{n} / \mathrm{a}$ \\
\hline \% BNF maximum antipsychotic dose (admission $v$. discharge) & $86.36 \pm 52.33$ & $72.40 \pm 43.78$ & $P=0.0048$ & $\mathrm{n} / \mathrm{a}$ \\
\hline Clozapine prescription (admission v. discharge) (n, \%) & $25(18)$ & $95(66)$ & $P<0.0001$ & $\mathrm{n} / \mathrm{a}$ \\
\hline
\end{tabular}



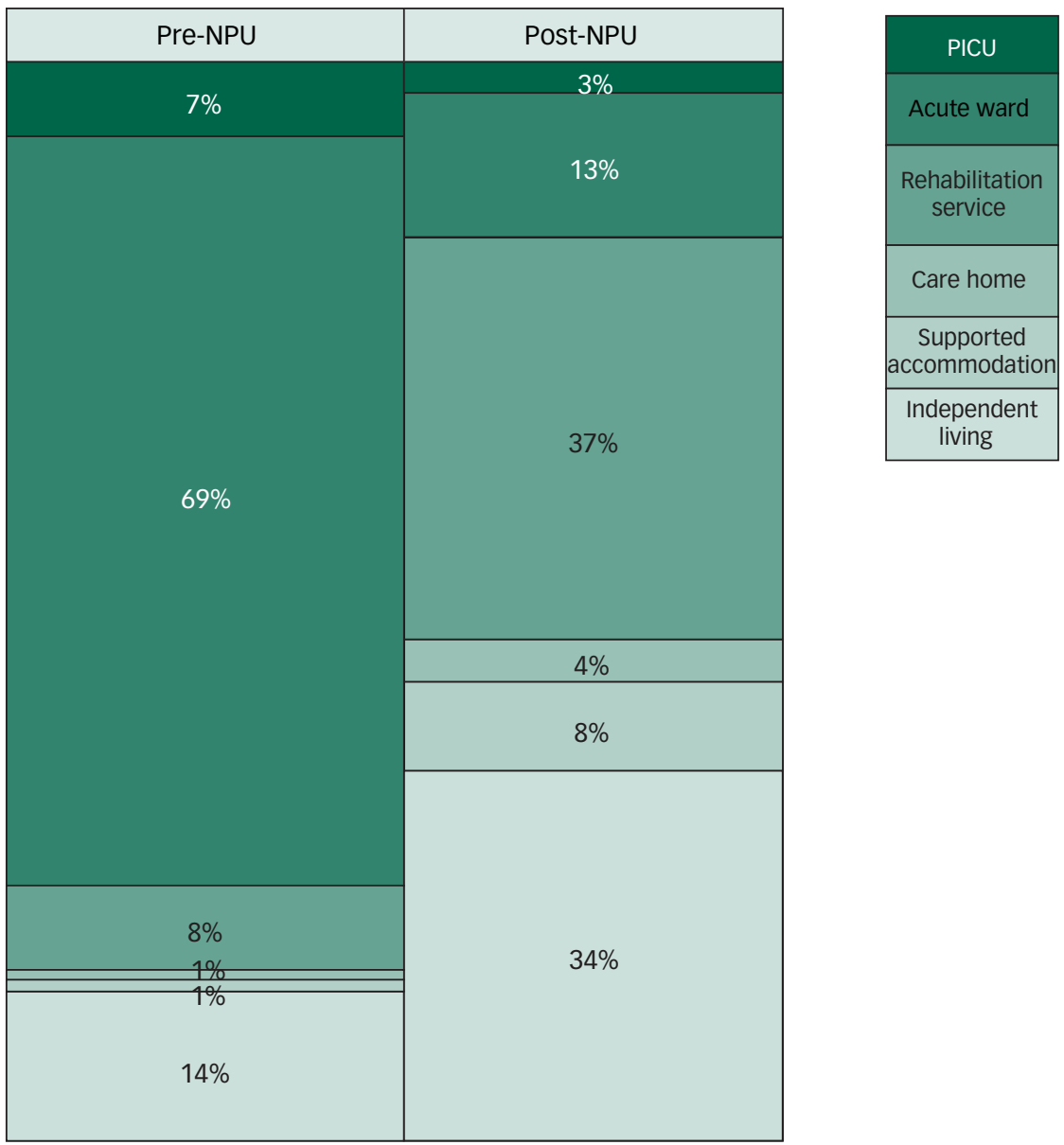

Fig. 3 Mosaic plot of treatments pre- and post-NPU placement.

expertise and have complex diagnostic and therapeutic capabilities which improve the chances of achieving a positive outcome. However, no specific guidelines exist on referring a patient with a treatment-refractory psychotic illness to a tertiary facility, as opposed to other complex chronic conditions such as epilepsy. ${ }^{28}$ Emerging data from a range of specialised NHS services have demonstrated improvement in patients' outcomes compared with non-specialist services. ${ }^{29-31}$ Such data re-emphasise the need for increased investment in specialist services in the NHS to continue to improve the population's health status and quality of care. $^{32}$

A significant overall reduction in the acuity of the care setting following discharge was demonstrated. Most of the patients referred to the NPU came from either PICUs or acute wards (76\%), whereas only $16 \%$ of the patients were discharged to such environments, with $37 \%$ moving on to rehabilitation services and $34 \%$ to independent accommodation in the community. Not only does this reflect a substantial improvement in patients' disability, it also corresponds to a significant cost reduction for health and social care systems, which is likely to be sustained well beyond the 2 years of follow-up demonstrated in this study, as $13 \%$ of people admitted to NPU had been in-patients consistently for at least 2 years before admission. Of note, the discharge of some of the patients to local acute wards might have been due to failure to find a placement best suited to their needs rather than the necessity of such a high level of support.

It is notable that these improvements in function were achieved despite a statistically significant reduction in total antipsychotic dose. Clozapine was introduced in most patients admitted to the NPU, as it is the gold standard treatment for TRS. This reflects the fact that many patients are referred to the NPU specifically for clozapine treatment, when this has been difficult to achieve in the acute setting, or when clozapine has been discontinued owing to significant concerns about physical health and a safe re-challenge of clozapine is sought. Our previous short-term outcome studies also reported a rationalisation of antipsychotic medication and increase in the use of clozapine from admission to discharge. ${ }^{21,22,33}$ Evidence suggests a response rate of up to $75 \%$ to clozapine in those who have failed to respond to previous antipsychotic trials. ${ }^{34}$ Furthermore, many patients with a diagnosis of a treatment-resistant psychotic disorder might decline oral medications owing to their delusional beliefs and lack of insight into their illness. ${ }^{35}$ The present study suggests that the level of support and expertise a tertiary service can provide allows patients to access clozapine where this had not proved possible in the local setting.

As mentioned above, patients are referred to the NPU from all over the UK. Consistently, our sample provides a good representation of patients with TRS in the UK, as demonstrated by the proportion of patients of Black African and Caribbean ethnicity, who represent approximately $3 \%$ of the UK population ${ }^{36}$ and have a 5.8 -fold increase in risk of schizophrenia compared with the White population. ${ }^{37}$ This reflects the nature of a national service which is open to referrals for anyone in the country who needs specialised care. Nonetheless, it does not reflect the reported disparity of involuntary psychiatric care in Black ethnic groups compared with White British patients. ${ }^{38,39}$ This might be owing to less inequality in tertiary referrals compared with compulsory in-patient psychiatric care, or it might reflect potential biases in the referral 
processes such that members of Black ethnic groups are less likely to be referred to specialised care than their White peers.

\section{Limitations}

A benefit of this study is the fact that informed consent was not required as the data were retrieved from pseudonymised databases. This eliminated the selection bias in favour of higher-functioning patients that often taints research on treatment-resistant psychosis. That said, this study was not without its limitations.

One potential limitation was that we had limited clinical information about the patients before admission and after discharge from the NPU, as their care was usually given by different healthcare providers. Instead, we considered hospital admission as a marker of the overall mental health of the patients. It may be argued that the change in services during the timeframe covered by our study could have influenced our results. Although this should be highlighted as a limitation, there were no major changes to TRS-focused service provision in SLaM in the timeframe considered.

The mirror-image design allowed for a within-patient analysis, minimising the selection bias that may complicate comparisons between groups in naturalistic research. Nonetheless, the lack of a comparator group is a potential disadvantage, and our results may reflect background variations occurring irrespective of the treatment received.

To obtain information about admissions, we used the HES database, which contains data on admissions within the NHS, including private patients treated in NHS hospitals, and care delivered by treatment centres (including those in the independent sector) funded by the NHS in England. Owing to administrative differences, admissions in Scotland, Wales, Northern Ireland and other areas of the British Isles are not recorded. Reassuringly, when sensitivity analyses were conducted including only patients residing in England $(n=140)$, no difference in significance was found for any of the variables considered.

Owing to the nature of this study, we could not focus on the patients' perspective or whether there was a specific component or components that yielded the positive outcome. However, there are some preliminary data which illustrate the aspects that families have found helpful during NPU admission. ${ }^{40}$

\section{Conclusions}

Our study demonstrates the long-term effectiveness of a tertiary service specialising in treatment-resistant psychotic disorders. This supports the existing literature on the need for and importance of specialist care for complex cases of severe mental illnesses.

Cecilia Casetta (D), King's College London, Department of Psychosis Studies, Institute of Psychiatry, Psychology \& Neuroscience, South London and Maudsley NHS Foundation Trust, London, UK; Fiona Gaughran, King's College London, Department of Psychosis Studies, Institute of Psychiatry, Psychology \& Neuroscience, South London and Maudsley NHS Foundation Trust, London, UK; Ebenezer Oloyede, King's College London, Department of Psychosis Studies, Institute of Psychiatry, Psychology \& Neuroscience, South London and Maudsley NHS Foundation Trust, London, UK; Juliana Onwumere, King's College London, Department of Psychology, Institute of Psychiatry, Psychology \& Neuroscience, South London and Maudsley NHS Foundation Trust, London, UK Megan Pritchard, NIHR Biomedical Research Centre for Mental Health; Sukhi S. Shergill, King's College London, Department of Psychosis Studies, Institute of Psychiatry, Psychology \& Neuroscience, South London and Maudsley NHS Foundation Trust, London, UK; Eromona Whiskey, King's College London, Department of Psychosis Studies, Institute of Psychiatry, Psychology \& Neuroscience, South London and Maudsley NHS Foundation Trust, London, UK; James Hunter MacCabe $\mathbb{D}^{\mathbb{D}}$, King's College London, Department of Psychosis Studies, Institute of Psychiatry, Psychology \& Neuroscience, South London and Maudsley NHS Foundation Trust, London, UK

Correspondence: Cecilia Casetta. Email: cecilia.casetta@gmail.com

First received 27 Feb 2020, final revision 14 May 2020, accepted 31 May 2020

\section{Acknowledgement}

The publication of this paper is supported by a grant from The Royal College of Psychiatrists Academic Freedom Fund established by Kenneth R. Kaufman, MD FRCPsych. For further details
about the fund please visit: https://www.cambridge.org/core/journals/bjpsych-open/information/instructions-contributors.

\section{Author contributions}

C.C. and J.H.M. contributed to the conception and design of the study. C.C., E.O. and M.P. collected and analysed the data. F.G. E.W. S.S.S. and J.O took part in the interpretation of the data; and all authors contributed to the drafting and revision of the manuscript.

\section{Funding}

This paper describes independent research funded by the National Institute for Health Research (NIHR) Biomedical Research Centre at SLaM and King's College London. The views expressed are those of the author(s) and not necessarily those of the NHS, the NIHR or the Department of Health

\section{Declaration of interest}

None.

ICMJE forms are in the supplementary material, available online at https://doi.org/10.1192/ bjo.2020.51.

\section{References}

1 McGrath J, Saha S, Chant D, Welham J. Schizophrenia: a concise overview of incidence, prevalence, and mortality. Epidemiol Rev 2008; 30(1): 67-76.

2 Charlson FJ, Ferrari AJ, Santomauro DF, Diminic S, Stockings E, Scott JG, et al. Global epidemiology and burden of schizophrenia: findings from the global burden of disease study 2016. Schizophr Bull 2018; 44(6): 1195-203.

3 Lally J, Maccabe JH. Antipsychotic medication in schizophrenia: a review. $\mathrm{Br}$ Med Bull 2015; 114: 169-79.

4 Howes OD, McCutcheon R, Agid O, de Bartolomeis A, van Beveren NJM, Birnbaum ML, et al. Treatment-resistant schizophrenia: Treatment Response and Resistance in Psychosis (TRRIP) Working Group consensus guidelines on diagnosis and terminology. Am J Psychiatry 2017; 174(3): 216-29.

5 Horsdal HT, Wimberley T, Köhler-Forsberg O, Baandrup L, Gasse C. Association between global functioning at first schizophrenia diagnosis and treatment resistance. Early Interv Psychiatry 2018; 12(6): 1198-202.

6 Gudeman JE, Shore MF. Beyond deinstitutionalization, a new class of facilities for the mentally ill. N Engl J Med 1984; 311(13): 832-6.

7 National Institute for Health and Care Excellence. Glossary. NICE, 2020 (https:// www.nice.org.uk/glossary?letter=t).

8 Ker S, Anderson I. Service innovations: developing a specialised (tertiary) service for the treatment of affective disorders. Psychiatr Bull 2006; 30: 103-5.

9 Wasylenki D, Goering P, Cochrane J, Durbin J, Rogers J, Prendergast P. Tertiary mental health services: I. Key concepts. Can J Psychiatry 2000; 45: 179-84.

10 Leucht S, Cipriani A, Spineli L, Mavridis D, Örey D, Richter F, et al. Comparative efficacy and tolerability of 15 antipsychotic drugs in schizophrenia: a multipletreatments meta-analysis. Lancet 2013; 382: 951-62.

11 Siskind D, Reddel T, MacCabe JH, Kisely S. The impact of clozapine initiation and cessation on psychiatric hospital admissions and bed days: a mirror image cohort study. Psychopharmacology 2019; 236(6): 1931-5.

12 Cho J, Hayes RD, Jewell A, Kadra G, Shetty H, MacCabe JH, et al. Clozapine and all-cause mortality in treatment-resistant schizophrenia: a historical cohort study. Acta Psychiatr Scand 2019; 139(3): 237-47.

13 Wimberley T, MacCabe JH, Laursen TM, Sørensen HJ, Astrup A, Horsdal HT, et al. Mortality and self-harm in association with clozapine in treatment-resistant schizophrenia. Am J Psychiatry 2017; 174(10): 990-8.

14 Patchan K, Vyas G, Hackman AL, Mackowick M, Richardson CM, Love RC, et al. Clozapine in reducing aggression and violence in forensic populations. Psychiatr Q 2018; 89(1): 157-68.

15 Bhavsar V, Kosidou K, Orsini N, Widman L, Maccabe J, Dalman C. Could clozapine reduce violent offending? Schizophr Bull 2018; 44: S67.

16 Volavka J. Aggression and substance abuse in schizophrenic patients. Psychiatry 1999; 60(suppl 12): 43-6.

17 Stroup TS, Lieberman JA, McEvoy JP, Davis SM, Swartz MS, Keefe RS, et al. CATIE Investigators. Results of phase 3 of the CATIE schizophrenia trial. Schizophr Res 2009; 107(1): 1-12

18 Thompson JV, Clark JM, Legge SE, Kadra G, Downs J, Walters JTR, et al Antipsychotic polypharmacy and augmentation strategies prior to clozapine 
initiation: a historical cohort study of 310 adults with treatment-resistant schizophrenic disorders. J Psychopharmacol 2016; 30(5): 436-43.

19 Dold M, Leucht S. Pharmacotherapy of treatment-resistant schizophrenia: a clinical perspective. Evid Based Ment Health 2014; 17(2): 33-7.

20 Kadra G, Stewart R, Shetty H, MacCabe JH, Chang CK, Kesserwani J, et al. Antipsychotic polypharmacy prescribing and risk of hospital readmission. Psychopharmacology 2018; 235(1): 281-9.

21 Perera G, Broadbent M, Callard F, Chang CK, Downs J, Dutta R, et al. Cohort profile of the South London and Maudsley NHS Foundation Trust Biomedica Research Centre (SLaM BRC) case register: current status and recent enhancement of an electronic mental health record-derived data resource. BMJ Open 2016; 6(3): e008721

22 Krivoy A, Joyce D, Tracy D, Gaughran F, MacCabe J, Lally J, et al. Real-world out comes in the management of refractory psychosis. J Clin Psychiatry 2019; 80 (5): $18 \mathrm{~m} 12716$.

23 Sarkar SN, Tracy DK, Fernandez MJ, Nalesnik N, Dhillon G, Onwumere J, et al. Unheard voices: outcomes of tertiary care for treatment-refractory psychosis. Psychiatr Bull 2014; 38(2): 71-4.

24 Joint Formulary Committee. British National Formulary (76th ed). BMJ Group and Pharmaceutical Press, 2018.

25 Shepard DJ, Insole LM, McAllister-Williams RH, Nicole FI. Are specialised affective disorder services useful? Psychiatr Bull 2009; 33: 41-3.

26 Ker S, Anderson I. Service innovations: developing a specialised (tertiary) service for the treatment of affective disorders. Psychiatr Bull 2006; 30: 103-5.

27 Brodaty $\mathrm{H}$, Harris L, Wilhelm K, Hickie I, Boyce P, Mitchell P, et al. Lessons from a mood disorders unit. Aust N Z J Psychiatry 1993; 27(2): 254-63.

28 National Institute for Health and Care Excellence. Epilepsies: diagnosis and management. NICE, 2013 (https://www.nice.org.uk/guidance/cg137/chapter/ 1-Guidance\#referral-for-complex-or-refractory-epilepsy (last updated: 11 February 2020))

29 Kingsmore $D$, Hole D, Gillis C. Why does specialist treatment of breast cancer improve survival? The role of surgical management. Br J Cancer 2004; 90 : $1920-5$.

30 Ismail F, Mitchell L, Casabonne D, Gulati A, Newton R, Proby CM, et al. Specialist dermatology clinics for organ transplant recipients significantly improve compliance with photoprotection and levels of skin cancer awareness. $\mathrm{Br} J$ Dermatol 2006; 155(5): 916-25.
31 Bird GT, Farquhar-Smith P, Wigmore T, Potter M, Gruber PC. Outcomes and prognostic factors in patients with haematological malignancy admitted to a specialist cancer intensive care unit: a 5 yr study. Br J Anaesth 2012; 108(3): 452-9.

32 Majeed A, Allwood D, Foley K, Bindman A. Healthcare outcomes and quality in the NHS: how do we compare and how might the NHS improve? BMJ 2018; 362: k3036.

33 Tracy DK, Joyce DW, Sarkar SN, Fernandez MM, Shergill SS. Skating on thin ice: pragmatic prescribing for medication refractory schizophrenia. BMC Psychiatry 2015; 15: 174.

34 Agid O, Arenovich T, Sajeev G, Zipursky RB, Kapur S, Foussias G, et al. An algorithm-based approach to first-episode schizophrenia: response rates over 3 prospective antipsychotic trials with a retrospective data analysis. J Clin Psychiatry 2011; 72(11): 1439-44.

35 Daod E, Krivoy A, Shoval G, Zubedat S, Lally J, Vadas L, et al. Psychiatrists' attitude towards the use of clozapine in the treatment of refractory schizophrenia: a nationwide survey. Psychiatry Res 2019; 275: 155-61.

36 Office for National Statistics. National Records of Scotland; Northern Ireland Statistics and Research Agency (2016): 2011 Census aggregate data. UK Data Service (Edition: June 2016). ONS, June 2016 (http://doi.org/10.5257/census/ aggregate-2011-1).

37 Fearon $\mathrm{P}$, Kirkbride J, Morgan $\mathrm{C}$, et al. Incidence of schizophrenia and other psychoses in ethnic minority groups: results from the MRC AESOP study. Psychol Med 2006; 36(11): 1541-50.

38 Barnett $\mathrm{P}$, Mackay E, Matthews H, Gate R, Greenwood H, Ariyo K, et al. Ethnic variations in compulsory detention under the Mental Health Act: a systematic review and meta-analysis of international data. Lancet Psychiatry 2019; 6(4): 305-17.

39 NHS Digital. Mental Health Bulletin 2017-18 Annual Report. NHS Digital, 2018 (https://digital.nhs.uk/data-and-information/publications/statistical/mentalhealth-bulletin/2017-18-annual-report).

40 Onwumere J, Jansen JE, Kuipers E. Editorial: family interventions in psychosis change outcomes in early intervention settings - how much does the evidence support this? Front Psychol 2018; 9: 406.

OPEN

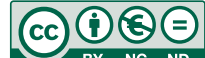

